\title{
Weak convergence of a modified subgradient extragradient algorithm for monotone variational inequalities in Banach spaces
}

\author{
Ying Liu*, Hang Kong \\ College of Mathematics and Information Science, Hebei University, Baoding, Hebei, 071002, China.
}

Communicated by S. S. Chang

\begin{abstract}
Applying the generalized projection operator, we introduce a modified subgradient extragradient algorithm in Banach spaces for a variational inequality involving a monotone Lipschitz continuous mapping which is more general than an inversestrongly-monotone mapping. Weak convergence of the iterative algorithm is also proved. An advantage of the algorithm is the computation of only one value of the inequality mapping and one projection onto the admissible set per one iteration. (C)2017 All rights reserved.
\end{abstract}

Keywords: Subgradient extragradient method, generalized projection operator, monotone mapping, variational inequality, Lipschitz continuous, weakly sequentially continuous.

2010 MSC: 47H09, 47H05, 47H06, 47J25, 47J05.

\section{Introduction}

Let $E$ be a real Banach space with norm $\|\cdot\|$, and $E^{*}$ be the dual of $E .\langle x, f\rangle$ denotes the duality pairing of $E$ and $E^{*}$. Suppose that $C$ is a nonempty, closed, and convex subset of $E$. In this paper, we study the problem of finding a point $u \in C$ such that

$$
\langle v-u, A u\rangle \geqslant 0, \quad \forall v \in C,
$$

where $A: C \rightarrow E^{*}$ is a single-valued mapping. This problem is called the variational inequality problem (VIP). The set of solutions of the variational inequality problem is denoted by $\mathrm{VI}(C, A)$. Such a problem is connected with the convex minimization problem, the complementarity problem, the problem of finding a point $u \in E$ satisfying $0=A u$ and so on. An operator $A$ of $C$ into $E^{*}$ is said to be

(i) monotone if $\langle x-y, A x-A y\rangle \geqslant 0$, for all $x, y \in C$;

(ii) $\alpha$-inverse-strongly-monotone if there exists a positive real number $\alpha$ such that

$$
\langle x-y, A x-A y\rangle \geqslant \alpha\|A x-A y\|^{2}, \quad \forall x, y \in C ;
$$

(iii) L-Lipschitz continuous if there exists a constant $\mathrm{L}>0$ such that

$$
\|A x-A y\| \leqslant L\|x-y\|, \quad \forall x, y \in C .
$$

\footnotetext{
*Corresponding author

Email addresses: 1y_cyh2013@163.com (Ying Liu), 1969849957@qq.com (Hang Kong)
} 
A great number of methods for solving variational inequality (1.1) have been proposed and analyzed by many authors in Hilbert spaces or Banach spaces when $A$ has monotonicity and Lipschitz continuity or inverse-strong-monotonicity, (see, for example, $[3,4,6,7,9-13,15,19,20,23]$ and the reference therein).

The simplest method is the gradient projection in which only one projection onto the feasible set $C$ is performed at each iteration, but this method requires the restrictive assumption that $A$ is strongly (or inverse strongly) monotone.

For example, Iiduka and Takahashi [11] proposed the following gradient projection method.

\section{Algorithm 1.1.}

$$
x_{n+1}=\Pi_{C} J^{-1}\left(J x_{n}-\lambda_{n} A x_{n}\right) .
$$

More precisely, they proved the following theorem.

Theorem 1.2. Let $\mathrm{E}$ be a 2-uniformly convex and uniformly smooth Banach space whose duality mapping $\mathrm{J}$ is weakly sequentially continuous, and $\mathrm{C}$ be a nonempty, closed convex subset of $\mathrm{E}$. Assume $\mathrm{A}: \mathrm{C} \rightarrow \mathrm{E}^{*}$ is an $\alpha$-inverse-strongly-monotone mapping satisfying the following condition

$$
\|A y\| \leqslant\|A y-A u\| \text { for all } y \in C \text { and } u \in V I(C, A) \text {. }
$$

If $\operatorname{VI}(\mathrm{C}, \mathrm{A}) \neq \emptyset$, and $\left\{\lambda_{\mathrm{n}}\right\} \subset[\mathrm{a}, \mathrm{b}]$ for some $\mathrm{a}, \mathrm{b}$ with $0<\mathrm{a}<\mathrm{b}<\mathrm{c}_{1} \alpha$, where $\mathrm{c}_{1}$ is the 2-uniformly convex constant, then the iterative sequence $\left\{x_{n}\right\}$ generated by (1.2) converges weakly to some element $z$ in $\operatorname{VI}(C, A)$. Further $z=\lim _{n \rightarrow \infty} \Pi_{V I(C, A)}\left(x_{n}\right)$.

We note that if $A$ is $\alpha$-inverse-strongly-monotone, then it is monotone and $\frac{1}{\alpha}$-Lipschitz continuous. But, the converse is not true. One problem arises naturally:

(P1) How to relax the inverse-strong monotonicity of $A$ to the monotonicity and Lipschitz continuity?

In addition, we also note that the condition (A) is not easy to be verified because $\operatorname{VI}(C, A)$ is not explicit. The necessity of the condition needs to be checked. Hence, we have the following problem.

(P2) Can the condition (A) be removed?

In 1976, Korpelevich [12] introduced the extragradient method for the saddle point problem and then, it was extended to VIP which only requires the monotonicity and the Lipschitz continuity of A,

$$
\left\{\begin{array}{l}
x_{0}=x \in C \\
y_{n}=P_{C}\left(x_{n}-\lambda A x_{n}\right), \\
x_{n+1}=P_{C}\left(x_{n}-\lambda A y_{n}\right) .
\end{array}\right.
$$

In this method, instead of a projection, she implemented two projections onto the feasible set $C$. In recent years, the extragradient method has been widely studied and modified by many authors via different ways, see for instance $[4-6,8,13,19,25]$ and the references therein.

In the next, we always let $\mathbb{H}$ be a Hilbert space and $\mathbb{N}$ be the set of all positive integers.

One of the drawbacks of the extragradient method is the necessity of two projections onto the admissible set $C$ in each iterate. It is not easy to compute when the structure of the set $C$ is complicated. As mentioned in [6], this may affect the efficiency of the method. Therefore, Censor et al. in [6] modified the the extragradient method and proposed the following iterative algorithm:

$$
\left\{\begin{array}{l}
x_{0} \in \mathbb{H} \\
y_{n}=P_{C}\left(x_{n}-\tau A\left(x_{n}\right)\right) \\
T_{n}=\left\{w \in \mathbb{H}:\left\langle x_{n}-\tau A\left(x_{n}\right)-y_{n}, w-y_{n}\right\rangle \leqslant 0\right\} \\
x_{n+1}=P_{T_{n}}\left(x_{n}-\tau A\left(y_{n}\right)\right) .
\end{array}\right.
$$

Method (1.4) replaces the second projection onto the closed and convex subset $C$ in (1.3) with the one onto the subgradient half-space $T_{n}$. So the method (1.4) is called the subgradient extragradient method. 
We note that, the set $T_{n}$ is a half-space, and hence (1.4) is easier to execute than (1.3). Under some mild assumptions, Censor et al. in [6] proved the method (1.4) converges weakly to a solution of variational inequality (1.1) in a Hilbert space.

The method (1.4) has been further modified and extended to obtain strong convergence results in Hilbert spaces and Banach spaces, see $[5,13,16]$ and the reference therein. In [13], the authors combined the method (1.4) with the Halpern method to propose the Halpern subgradient extragradient method for the strong convergence in Hilbert spaces. Recently, Liu [16] extended the Halpern subgradient extragradient method to Banach spaces and constructed the following algorithm.

For any $x_{0} \in E$, define a sequence $\left\{x_{n}\right\}$ iteratively by the following:

\section{Algorithm 1.3.}

$$
\left\{\begin{array}{l}
y_{n}=\Pi_{C} J^{-1}\left(J x_{n}-\lambda_{n} A\left(x_{n}\right)\right) \\
T_{n}=\left\{w \in E:\left\langle w-y_{n}, J x_{n}-\lambda_{n} A\left(x_{n}\right)-J y_{n}\right\rangle \leqslant 0\right\} \\
w_{n}=\Pi_{T_{n}} J^{-1}\left(J x_{n}-\lambda_{n} A\left(y_{n}\right)\right) \\
x_{n+1}=J^{-1}\left(\alpha_{n} J x_{0}+\left(1-\alpha_{n}\right) J w_{n}\right)
\end{array}\right.
$$

where $\left\{\alpha_{n}\right\} \subset[0,1]$ satisfying $\lim _{n \rightarrow \infty} \alpha_{n}=0$ and $\sum_{n=1}^{\infty} \alpha_{n}=\infty$ and $\left\{\lambda_{n}\right\} \subset(0, \infty)$ satisfying $0<\inf _{n \geqslant 1} \lambda_{n} \leqslant$ $\sup \lambda_{n}<\frac{c_{1}}{L}$. Liu in [16] proved the iterative sequence $\left\{x_{n}\right\}$ generated by Algorithm 1.3 strongly converges $n \geqslant 1$

to an element of $\mathrm{VI}(\mathrm{C}, \mathrm{A})$ under conditions (C1)-(C3) of Section 3 of this paper.

But, the drawback of the subgradient extragradient method is the computation of values of the mapping $A$ at two different points to pass to the next iteration. Very often variational inequality problems which arise from optimal control, provide a very complicated operator such that only computation of the values of the mapping $A$ is a very sophisticated problem (for more details see [14]).

To avoid the drawback, Malitsky and Semenov [17] combined the Popov algorithm [21] and the subgradient extragradient method [6] to propose the following iterative algorithm:

\section{Algorithm 1.4.}

1. Specify $x_{0}, y_{0} \in C$, and $\lambda>0$.

2. Compute

$$
\left\{\begin{array}{l}
x_{1}=P_{C}\left(x_{0}-\lambda A\left(y_{0}\right)\right) \\
y_{1}=P_{C}\left(x_{1}-\lambda A\left(y_{0}\right)\right)
\end{array}\right.
$$

3. Given $x_{n}, y_{n}$ and $y_{n-1}$, construct a half-space

$$
H_{n}=\left\{z \in \mathbb{H}:\left\langle x_{n}-\lambda A y_{n-1}-y_{n}, z-y_{n}\right\rangle \leqslant 0\right\}
$$

and compute

$$
\left\{\begin{array}{l}
x_{n+1}=P_{H_{n}}\left(x_{n}-\lambda A\left(y_{n}\right)\right) \\
y_{n+1}=P_{C}\left(x_{n+1}-\lambda A\left(y_{n}\right)\right)
\end{array}\right.
$$

4. If $x_{n+1}=x_{n}$ and $y_{n+1}=y_{n}=y_{n-1}$, then complete the computation and, otherwise, put $n:=n+1$ and pass to step 3 .

More precisely, they proved the following theorem.

Theorem 1.5. Let $\mathrm{C}$ be a nonempty closed and convex subset of a Hilbert space $\mathbb{H}$. Let $\mathrm{A}: \mathrm{C} \rightarrow \mathbb{H}$ be monotone and Lipschitz with a constant $\mathrm{L}>0$. Let $\mathrm{VI}(\mathrm{C}, \mathrm{A}) \neq \emptyset$ and $\lambda \in\left(0, \frac{1}{3 \mathrm{~L}}\right)$. Then sequences $\left\{x_{n}\right\}$ and $\left\{\mathrm{y}_{\mathrm{n}}\right\}$ generated by Algorithm 1.4 weakly converge to some point $z \in \mathrm{VI}(\mathrm{C}, \mathrm{A})$.

We note that the operator $A$ in Algorithm 1.4 is only monotone and Lipschitz continuous and does not require condition (A) assumed in [11]. Furthermore, Algorithm 1.4 needs only one computation of projection onto the admissible set $C$ and only one value of $A$ on every iteration. But, we observe that the convergence result of Algorithm 1.4 is valid only in Hilbert spaces. However, many important problems related to practical problems are generally defined in Banach spaces. This now leads to the following 
important problem.

(P3) How to extend Algorithm 1.4 to the more general Banach spaces?

The purpose of this paper is to construct a new method for finding an element of $\operatorname{VI}(C, A)$ for a Lipschitz-continuous, monotone mapping in a 2-uniformly convex and uniformly smooth Banach space. Consequently, we will solve problems (P1)-(P3).

The paper is organized as follows. In the next section we recall some definitions and preliminary results used in the paper. Section 3 presents our algorithm and deals with the convergence analysis. In Section 4, we give two numerical experiments to illustrate the proposed algorithm in comparison with the Algorithm 1.3 and in Section 5 we offer concluding comments.

\section{Preliminaries}

When $\left\{x_{n}\right\}$ is a sequence in $E$, we denote the strong convergence of $\left\{x_{n}\right\}$ to $x \in E$ by $x_{n} \rightarrow x$ and the weak convergence by $x_{n} \rightarrow x$.

Let $U=\{x \in E:\|x\|=1\}$. A Banach space $E$ is said to be strictly convex if for any $x, y \in U$ and $x \neq y$ implies $\left\|\frac{x+y}{2}\right\|<1$. It is also said to be uniformly convex if for each $\epsilon \in(0,2]$, there exists $\delta>0$ such that for any $x, y \in U,\|x-y\| \geqslant \epsilon$ implies $\left\|\frac{x+y}{2}\right\| \leqslant 1-\delta$. It is known that a uniformly convex Banach space is reflexive and strictly convex. We define a function $\delta:[0,2] \rightarrow[0,1]$ called the modulus of convexity of $E$ as follows:

$$
\delta(\epsilon)=\inf \left\{1-\left\|\frac{x+y}{2}\right\|: x, y \in U,\|x-y\| \geqslant \epsilon\right\} .
$$

Then $E$ is uniformly convex if and only if $\delta(\epsilon)>0$ for all $\epsilon \in(0,2]$. Let $p$ be a fixed real number with $p \geqslant 2$. A Banach space $E$ is said to be p-uniformly convex if there exists a constant $c>0$ such that $\delta(\epsilon) \geqslant c \epsilon^{p}$ for all $\epsilon \in[0,2]$. It is obvious that a p-uniformly convex Banach space is uniformly convex. For example, see [2] and [22] for more details. A Banach space $E$ is said to be smooth if the $\operatorname{limit}_{t \rightarrow 0} \frac{\|x+t y\|-\|x\|}{t}$ exists for all $x, y \in U$. It is also said to be uniformly smooth if the limit is attained uniformly for $x, y \in U$. It is well-known that Hilbert and the Lebesgue $\mathrm{L}^{\mathrm{q}}(1<\mathrm{q} \leqslant 2)$ spaces are 2-uniformly convex, uniformly smooth.

Let $\mathrm{J}: \mathrm{E} \rightarrow 2^{\mathrm{E}^{*}}$ be the normalized duality mapping defined by

$$
\mathrm{Jx}:=\left\{v \in \mathrm{E}^{*}:\langle x, v\rangle=\|v\|^{2}=\|x\|^{2}\right\}, \quad \forall x \in \mathrm{E} .
$$

The following properties of the duality mapping J can be found in [1].

(i) If $E$ is smooth, then $J$ is single-valued.

(ii) If $E$ is strictly convex, then $J$ is one-to-one and strictly monotone.

(iii) If $E$ is reflexive, then $J$ is surjective.

(iv) If $E$ is uniformly smooth, then $J$ is uniformly norm-to-norm continuous on each bounded subset of E.

(v) If $E$ is a smooth, strictly convex, and reflexive Banach space, then J is single-valued, one-to-one, and onto. Simultaneously, $\mathrm{J}^{-1}$ is also single-valued, one-to-one and onto, and it is the duality mapping from $E^{*}$ to $E$.

Let $\mathrm{E}$ be a smooth Banach space. We know the following Lyapunov functional introduced by $\mathrm{Al}^{\prime}$ ber and Reich [1]:

$$
\phi(x, y)=\|x\|^{2}-2\langle x, J y\rangle+\|y\|^{2}, \quad \forall x, y \in E .
$$

Clearly, we have from the definition of $\phi$ that

(B1) $(\|x\|-\|y\|)^{2} \leqslant \phi(y, x) \leqslant(\|x\|+\|y\|)^{2}$; 
(B2) $\phi(x, y)=\phi(x, z)+\phi(z, y)+2\langle x-z, J z-J y\rangle$;

(B3) $\phi(x, y)=\langle x, J x-J y\rangle+\langle y-x, J y\rangle \leqslant\|x\|\|J x-J y\|+\|y-x\|\|y\|$.

Lemma 2.1 ([18]). Let $\mathrm{E}$ be a uniformly convex and smooth Banach space and let $\left\{\mathrm{y}_{\mathrm{n}}\right\},\left\{z_{\mathrm{n}}\right\}$ be two sequences of $\mathrm{E}$. If $\phi\left(y_{n}, z_{n}\right) \rightarrow 0$, and either $\left\{y_{n}\right\}$ or $\left\{z_{n}\right\}$ is bounded, then $y_{n}-z_{n} \rightarrow 0$.

Let $E$ be a reflexive, strictly convex, and smooth Banach space. $C$ denotes a nonempty, closed and convex subset of $\mathrm{E}$. By Al'ber and Reich [1], for each $x \in \mathrm{E}$, there exists a unique element $\mathrm{x}_{0} \in \mathrm{C}$ (denoted by $\Pi_{C}(x)$ ) such that

$$
\phi\left(x_{0}, x\right)=\min _{y \in C} \phi(y, x) .
$$

The mapping $\Pi_{C}: E \rightarrow C$, defined by $\Pi_{C}(x)=x_{0}$, is called the generalized projection operator from $E$ onto $C$. Moreover, $x_{0}$ is called the generalized projection of $x$. In a Hilbert space, $\Pi_{C}=P_{C}$ (the metric projection operator).

Lemma 2.2 ([11, 18]). Let $\mathrm{C}$ be a nonempty closed and convex subset of a smooth Banach space $\mathrm{E}$ and $\mathrm{x} \in \mathrm{E}$. Then, $x_{0}=\Pi_{C} x$ if and only if

$$
\left\langle x_{0}-\mathrm{y}, \mathrm{Jx}-\mathrm{J} \mathrm{x}_{0}\right\rangle \geqslant 0, \quad \forall \mathrm{y} \in \mathrm{C} .
$$

Lemma $2.3([11,18])$. Let $\mathrm{E}$ be a reflexive, strictly convex, and smooth Banach space, let $\mathrm{C}$ be a nonempty closed and convex subset of $\mathrm{E}$ and let $\mathrm{x} \in \mathrm{E}$. Then

$$
\phi\left(y, \Pi_{C} x\right)+\phi\left(\Pi_{C} x, x\right) \leqslant \phi(y, x), \quad \forall y \in C .
$$

Lemma 2.4 ([11]). Let $\mathrm{C}$ be a nonempty, closed and convex subset of a Banach space $\mathrm{E}$ and $\mathrm{A}$ a monotone, hemicontinuous operator of $\mathrm{C}$ into $\mathrm{E}^{*}$. Then

$$
\operatorname{VI}(C, A)=\{u \in C:\langle v-u, A v\rangle \geqslant 0 \text { for all } v \in C\} .
$$

It is obvious from Lemma 2.4 that the set $\operatorname{VI}(C, A)$ is a closed and convex subset of $C$.

Lemma 2.5 ([20]). Let $\mathrm{E}$ be a 2-uniformly convex and smooth Banach space. Then, for every $x, y \in E, \phi(x, y) \geqslant$ $c_{1}\|x-y\|^{2}$, where $c_{1}>0$ is the 2-uniformly convexity constant of $\mathrm{E}$.

Lemma 2.6 ([24]). Let $\left\{a_{n}\right\}$ and $\left\{t_{n}\right\}$ be two sequences of nonnegative real numbers satisfying the inequality $a_{n+1} \leqslant a_{n}+t_{n}$ for all $n \in \mathbb{N}$. If $\sum_{n=1}^{\infty} t_{n}<\infty$, then $\lim _{n \rightarrow \infty} a_{n}$ exists.

\section{Main results}

Inspired by the results of [11, 17], we propose the following Algorithm 3.1 to extend Algorithm 1.4 from Hilbert spaces to Banach spaces and prove a weak convergence theorem.

In this section, we always assume the following conditions.

(C1) E is a 2-uniformly convex and uniformly smooth Banach space with the 2-uniformly convexity constant $c_{1}$ and $C$ is a nonempty, closed convex subset of $E$.

(C2) The mapping $A: C \rightarrow E^{*}$ is monotone and Lipschitz continuous with Lipschitz constant $L>0$.

(C3) $\operatorname{VI}(C, A) \neq \emptyset$.

\section{Algorithm 3.1.}

1. Specify $x_{0}, y_{0} \in C$, and $\lambda>0$.

2. Compute

$$
\left\{\begin{array}{l}
x_{1}=\Pi_{C} J^{-1}\left(J x_{0}-\lambda A\left(y_{0}\right)\right) \\
y_{1}=\Pi_{C} J^{-1}\left(J x_{1}-\lambda A\left(y_{0}\right)\right)
\end{array}\right.
$$


3. Given $x_{n}, y_{n}$ and $y_{n-1}$, construct a half-space

$$
H_{n}=\left\{z \in E:\left\langle z-y_{n}, J x_{n}-\lambda A y_{n-1}-J y_{n}\right\rangle \leqslant 0\right\}
$$

and compute

$$
\left\{\begin{array}{l}
x_{n+1}=\Pi_{H_{n}} J^{-1}\left(J x_{n}-\lambda A\left(y_{n}\right)\right) \\
y_{n+1}=\Pi_{C} J^{-1}\left(J x_{n+1}-\lambda A\left(y_{n}\right)\right)
\end{array}\right.
$$

4. If $x_{n+1}=x_{n}$ and $y_{n+1}=y_{n}=y_{n-1}$, then complete the computation and, otherwise, put $n:=n+1$ and pass to step 3.

Remark 3.2. It is obvious that $H_{n}$ is a half-space. Furthermore, since $y_{n}=\Pi_{C} J^{-1}\left(J x_{n}-\lambda A\left(y_{n-1}\right)\right)$, by Lemma 2.2 we have $C \subset H_{n}$ for every $n \in \mathbb{N}$.

First, we show that the stopping criterion in Algorithm 3.1 is valid.

Lemma 3.3. If $x_{n+1}=x_{n}$ and $y_{n+1}=y_{n}=y_{n-1}$ in Algorithm 3.1, then $y_{n} \in V I(C, A)$.

Proof. If we have $x_{n+1}=x_{n}$ in Algorithm 3.1, then Lemma 2.2 implies

$$
\left\langle x_{n}-x, \lambda A y_{n}\right\rangle \leqslant 0, \quad \forall x \in H_{n} .
$$

Taking into account that $x_{n+1} \in H_{n}$ and $y_{n}=y_{n-1}$, we obtain $\left\langle x_{n}-y_{n}, J x_{n}-\lambda A y_{n}-J y_{n}\right\rangle \leqslant 0$, whence we conclude that $\left\langle x_{n}-y_{n}, A y_{n}\right\rangle \geqslant 0$, since $J$ is strictly monotone. Then we represent inequality (3.1) in the form

$$
\left\langle x-y_{n}, A y_{n}\right\rangle-\left\langle x_{n}-y_{n}, A y_{n}\right\rangle \geqslant 0, \forall x \in H_{n}
$$

Hence, we have

$$
\left\langle x-y_{n}, A y_{n}\right\rangle \geqslant\left\langle x_{n}-y_{n}, A y_{n}\right\rangle \geqslant 0, \forall x \in H_{n}
$$

Since $y_{n} \in C \subset H_{n}$, we obtain that $y_{n} \in V I(C, A)$.

Next, we prove an important inequality relating the Lyapunov functional.

Lemma 3.4. Let $\left\{x_{n}\right\}$ and $\left\{y_{n}\right\}$ be generated by Algorithm 3.1. Then, under conditions (C1)-(C3), we have

$$
\phi\left(u, x_{n+1}\right) \leqslant \phi\left(u, x_{n}\right)-\left(1-\frac{2 \lambda L}{c_{1}}\right) \phi\left(x_{n+1}, y_{n}\right)-\left(1-\frac{\lambda L}{c_{1}}\right) \phi\left(y_{n}, x_{n}\right)+\frac{\lambda L}{c_{1}} \phi\left(x_{n}, y_{n-1}\right), \forall u \in V I(C, A) .
$$

Proof. Since $x_{n+1}=\Pi_{H_{n}} J^{-1}\left(J x_{n}-\lambda A\left(y_{n}\right)\right)$, it follows from Lemma 2.3, (B2), and $\operatorname{VI}(C, A) \subset C \subset H_{n}$ that, for any $u \in \operatorname{VI}(C, A)$,

$$
\begin{aligned}
\phi\left(u, x_{n+1}\right) \leqslant & \phi\left(u, J^{-1}\left(J x_{n}-\lambda A\left(y_{n}\right)\right)\right)-\phi\left(x_{n+1}, J^{-1}\left(J x_{n}-\lambda A\left(y_{n}\right)\right)\right) \\
= & \phi\left(u, x_{n}\right)+\phi\left(x_{n}, J^{-1}\left(J x_{n}-\lambda A\left(y_{n}\right)\right)\right)+2\left\langle u-x_{n}, \lambda A\left(y_{n}\right)\right\rangle \\
& -\phi\left(x_{n+1}, x_{n}\right)-\phi\left(x_{n}, J^{-1}\left(J x_{n}-\lambda A\left(y_{n}\right)\right)\right)-2\left\langle x_{n+1}-x_{n}, \lambda A\left(y_{n}\right)\right\rangle \\
= & \phi\left(u, x_{n}\right)-\phi\left(x_{n+1}, x_{n}\right)+2 \lambda\left\langle u-x_{n+1}, A\left(y_{n}\right)\right\rangle .
\end{aligned}
$$

Since $u \in \operatorname{VI}(C, A)$ and $A$ is monotone on $C$, we have

$$
\left\langle y_{n}-u, A\left(y_{n}\right)\right\rangle \geqslant 0 \text {. }
$$

Adding a nonnegative addend $2 \lambda\left\langle y_{n}-u, A\left(y_{n}\right)\right\rangle$ to the right side of inequality (3.3), we obtain

$$
\begin{aligned}
& \phi\left(u, x_{n+1}\right) \\
& \leqslant \phi\left(u, x_{n}\right)-\phi\left(x_{n+1}, x_{n}\right)+2 \lambda\left\langle y_{n}-x_{n+1}, A\left(y_{n}\right)\right\rangle \\
&= \phi\left(u, x_{n}\right)-\phi\left(x_{n+1}, y_{n}\right)-\phi\left(y_{n}, x_{n}\right)-2\left\langle x_{n+1}-y_{n}, J y_{n}-J x_{n}\right\rangle+2 \lambda\left\langle y_{n}-x_{n+1}, A\left(y_{n}\right)\right\rangle \\
&= \phi\left(u, x_{n}\right)-\phi\left(x_{n+1}, y_{n}\right)-\phi\left(y_{n}, x_{n}\right)+2\left\langle x_{n+1}-y_{n}, J x_{n}-J y_{n}-\lambda A\left(y_{n-1}\right)\right\rangle \\
&+2 \lambda\left\langle x_{n+1}-y_{n}, A y_{n-1}-A y_{n}\right\rangle .
\end{aligned}
$$


By the definition of $H_{n}$, we have

$$
\left\langle x_{n+1}-y_{n}, J x_{n}-\lambda A y_{n-1}-J y_{n}\right\rangle \leqslant 0 .
$$

We estimate the addend $2 \lambda\left\langle x_{n+1}-y_{n}, A y_{n-1}-A y_{n}\right\rangle$ in inequality (3.4) by the Lipschitz continuity of $A$, Lemma 2.5, and Cauchy-Schwarz inequality as follows:

$$
\begin{aligned}
2 \lambda\left\langle x_{n+1}-y_{n}, A y_{n-1}-A y_{n}\right\rangle & \leqslant 2 \lambda L\left\|x_{n+1}-y_{n}\right\|\left\|y_{n}-y_{n-1}\right\| \\
& \leqslant 2 \lambda L\left\|x_{n+1}-y_{n}\right\|\left(\left\|y_{n}-x_{n}\right\|+\left\|y_{n-1}-x_{n}\right\|\right) \\
& \leqslant \lambda L\left(\left\|y_{n}-x_{n}\right\|^{2}+2\left\|x_{n+1}-y_{n}\right\|^{2}+\left\|y_{n-1}-x_{n}\right\|^{2}\right) \\
& \leqslant \frac{\lambda L}{c_{1}}\left(\phi\left(y_{n}, x_{n}\right)+\phi\left(x_{n}, y_{n-1}\right)+2 \phi\left(x_{n+1}, y_{n}\right)\right) .
\end{aligned}
$$

Combining (3.4), (3.5) with (3.6), we have that (3.2) holds.

Theorem 3.5. Let the duality mapping J be weakly sequentially continuous and $\lambda \in\left(0, \frac{c_{1}}{3 \mathrm{~L}}\right)$. Then, under conditions (C1)-(C3), sequences $\left\{x_{n}\right\}$ and $\left\{y_{n}\right\}$ generated by Algorithm 3.1 weakly converge to some point $z \in V I(C, A)$. Furthermore, $z=\lim _{n \rightarrow \infty} \Pi_{\mathrm{VI}(\mathrm{C}, \mathrm{A})}\left(\mathrm{x}_{\mathrm{n}}\right)$.

Proof. We first show the boundedness of the sequence $\left\{x_{n}\right\}$. Summing up the inequalities (3.2) for all numbers $0,1,2, \ldots, n$, we obtain

$$
\begin{aligned}
\phi\left(u, x_{n+1}\right) \leqslant & \phi\left(u, x_{1}\right)-\left(1-\frac{2 \lambda L}{c_{1}}\right) \sum_{k=0}^{n-1} \phi\left(x_{n+1-k}, y_{n-k}\right) \\
& -\left(1-\frac{\lambda L}{c_{1}}\right) \sum_{k=0}^{n-1} \phi\left(y_{n-k}, x_{n-k}\right)+\frac{\lambda L}{c_{1}} \sum_{k=0}^{n-1} \phi\left(x_{n-k}, y_{n-k-1}\right) \\
= & \phi\left(u, x_{1}\right)-\left(1-\frac{3 \lambda L}{c_{1}}\right) \sum_{k=0}^{n-2} \phi\left(x_{n-k}, y_{n-k-1}\right)-\left(1-\frac{\lambda L}{c_{1}}\right) \sum_{k=0}^{n-1} \phi\left(y_{n-k}, x_{n-k}\right) \\
& +\frac{\lambda L}{c_{1}} \phi\left(x_{1}, y_{0}\right)-\left(1-\frac{2 \lambda L}{c_{1}}\right) \phi\left(x_{n+1}, y_{n}\right) \leqslant \phi\left(u, x_{1}\right)+\frac{\lambda L}{c_{1}} \phi\left(x_{1}, y_{0}\right) .
\end{aligned}
$$

This implies the boundedness of the sequence $\left\{x_{n}\right\}$. Let $n-k=m$, then, $\sum_{k=0}^{n-2} \phi\left(x_{n-k}, y_{n-k-1}\right)=$ $\sum_{m=2}^{n} \phi\left(x_{m}, y_{m-1}\right)$. In view of inequality (3.7), we have $\sum_{m=2}^{n} \phi\left(x_{m}, y_{m-1}\right)$ is bounded. This implies that the positive series $\sum_{n=1}^{\infty} \phi\left(x_{n}, y_{n-1}\right)$ is convergent. Similarly, we have that the positive series $\sum_{n=1}^{\infty} \phi\left(y_{n}, x_{n}\right)$ is also convergent. Thus, we have

$$
\lim _{n \rightarrow \infty} \phi\left(x_{n}, y_{n-1}\right)=\lim _{n \rightarrow \infty} \phi\left(y_{n}, x_{n}\right)=0 .
$$

By Lemma 2.1, we have

$$
\lim _{n \rightarrow \infty}\left\|x_{n}-y_{n-1}\right\|=\lim _{n \rightarrow \infty}\left\|y_{n}-x_{n}\right\|=0
$$

Since $\left\|y_{n}-y_{n-1}\right\| \leqslant\left\|y_{n}-x_{n}\right\|+\left\|x_{n}-y_{n-1}\right\|$, by (3.8), we have

$$
\lim _{n \rightarrow \infty}\left\|y_{n}-y_{n-1}\right\|=0 \text {. }
$$

Since J is uniformly norm-to-norm continuous on bounded sets, we have that

$$
\lim _{n \rightarrow \infty}\left\|J x_{n}-J y_{n-1}\right\|=\lim _{n \rightarrow \infty}\left\|J y_{n}-J x_{n}\right\|=0 .
$$


Let $\left\{x_{n_{k}}\right\}$ be a subsequence of $\left\{x_{n}\right\}$ such that $x_{n_{k}} \rightarrow z \in C$. Then, $y_{n_{k}} \rightarrow z \in C$. Now, we show that $z \in \operatorname{VI}(C, A)$. Since $y_{n+1}=\Pi_{C} J^{-1}\left(J x_{n+1}-\lambda A\left(y_{n}\right)\right)$, it follows from Lemma 2.2 that

$$
\left\langle x-y_{n_{k}+1}, J y_{n_{k}+1}-J x_{n_{k}+1}+\lambda A y_{n_{k}}\right\rangle \geqslant 0, \quad \forall x \in C .
$$

Using the monotonicity of the mapping $A$, we infer that

$$
\begin{aligned}
0 & \leqslant\left\langle x-y_{n_{k}+1}, J y_{n_{k}+1}-J x_{n_{k}+1}+\lambda A y_{n_{k}}\right\rangle \\
& =\left\langle x-y_{n_{k}+1}, J y_{n_{k}+1}-J x_{n_{k}+1}\right\rangle+\lambda\left\langle y_{n_{k}}-y_{n_{k}+1}, A y_{n_{k}}\right\rangle+\lambda\left\langle x-y_{n_{k}}, A y_{n_{k}}\right\rangle \\
& \leqslant\left\langle x-y_{n_{k}+1}, J y_{n_{k}+1}-J x_{n_{k}+1}\right\rangle+\lambda\left\langle y_{n_{k}}-y_{n_{k}+1}, A y_{n_{k}}\right\rangle+\lambda\left\langle x-y_{n_{k}}, A x\right\rangle .
\end{aligned}
$$

Passing to the limit with allowance for equalities (3.9) and (3.10), we obtain $\langle x-z, A x\rangle \geqslant 0$ for all $x \in C$. Hence, $z \in \operatorname{VI}(C, A)$ by Lemma 2.4 .

Let us show that $x_{n} \rightarrow z$. Define $u_{n}:=\Pi_{V I(C, A)} x_{n}$ for all $n \in \mathbb{N}$. Since $z \in \operatorname{VI}(C, A)$, we have $\phi\left(u_{n}, x_{n}\right) \leqslant \phi\left(z, x_{n}\right)$. Thus, $\left\{u_{n}\right\}$ is bounded. Next, we show $\left\{u_{n}\right\}$ is a Cauchy sequence. In fact, by Lemma 2.3 and inequality (3.2), we deduce that

$$
\phi\left(u_{n+1}, x_{n+1}\right) \leqslant \phi\left(u_{n}, x_{n+1}\right) \leqslant \phi\left(u_{n}, x_{n}\right)+\frac{\lambda L}{c_{1}} \phi\left(x_{n}, y_{n-1}\right) .
$$

The summability of the series $\sum_{n} \phi\left(x_{n}, y_{n-1}\right)$ and Lemma 2.6 imply the existence of $\lim _{n \rightarrow \infty} \phi\left(u_{n}, x_{n}\right)$. Using inequality (3.2), for all $m>n$, we have

$$
\phi\left(u_{n}, x_{m}\right) \leqslant \phi\left(u_{n}, x_{m-1}\right)+\frac{\lambda L}{c_{1}} \phi\left(x_{m-1}, y_{m-2}\right) \leqslant \cdots \leqslant \phi\left(u_{n}, x_{n}\right)+\frac{\lambda L}{c_{1}} \sum_{k=n}^{m-1} \phi\left(x_{k}, y_{k-1}\right) .
$$

Then we infer from $u_{m}=\Pi_{V I(, C, A)} x_{m}, u_{n}=\Pi_{V I(, C, A)} x_{n} \in V I(, C, A)$, Lemma 2.3, and (3.11) that

$$
\phi\left(u_{n}, u_{m}\right) \leqslant \phi\left(u_{n}, x_{m}\right)-\phi\left(u_{m}, x_{m}\right) \leqslant \phi\left(u_{n}, x_{n}\right)+\frac{\lambda L}{c_{1}} \sum_{k=n}^{m-1} \phi\left(x_{k}, y_{k-1}\right)-\phi\left(u_{m}, x_{m}\right)
$$

for all $m>n$. The existence of $\lim _{n \rightarrow \infty} \phi\left(u_{n}, x_{n}\right)$ and the summability of the series $\sum_{n} \phi\left(x_{n}, y_{n-1}\right)$ imply $\lim _{n \rightarrow \infty} \phi\left(u_{n}, u_{m}\right)=0$, and hence we have from Lemma 2.1 that $\lim _{n \rightarrow \infty}\left\|u_{n}-u_{m}\right\|=0$ for all $m>n$. Consequently, $\left\{u_{n}\right\}$ is a Cauchy sequence. Since $V I(C, A)$ is closed, we have that $\left\{u_{n}\right\}$ converges strongly to $\tilde{z} \in \operatorname{VI}(C, A)$. By the uniform smoothness of $E$, we also have that $\lim _{n \rightarrow \infty}\left\|J u_{n}-J \tilde{z}\right\|=0$. Now, we prove that $\tilde{z}=z$. In fact, it follows from Lemma 2.2, $u_{n}=\Pi_{V I(C, A)} x_{n}$, and $z \in V I(C, A)$ that $\left\langle z-u_{n_{k}}, J u_{n_{k}}-J x_{n_{k}}\right\rangle \geqslant$ 0 . By the weakly sequential continuity of $\mathrm{J}$, we infer that $\langle z-\tilde{z}, \mathrm{~J} \tilde{z}-\mathrm{J} z\rangle \geqslant 0$. Hence we have from the monotonicity of $J$ that $\langle z-\tilde{z}, J \tilde{z}-J z\rangle=0$. Since $E$ is strictly convex, we have that $\tilde{z}=z$. Therefore, the whole sequence $\left\{x_{n}\right\}$ converges weakly to $z=\lim _{n \rightarrow \infty} \Pi_{V I(C, A)} x_{n}$. Further, $\left\|x_{n}-y_{n}\right\| \rightarrow 0$ implies $\left\{y_{n}\right\}$ also converges weakly to $z=\lim _{n \rightarrow \infty} \Pi_{V I(C, A)} x_{n}$.

Remark 3.6. When $\mathrm{E}=\mathbb{H}$ (a Hilbert space), $\mathrm{c}_{1}=1, \Pi_{\mathrm{C}}=\mathrm{P}_{\mathrm{C}}$, and $\mathrm{J}=\mathrm{I}$, then, Theorem 3.5 reduces to Theorem 1.5. That is to say that Theorem 3.5 absolutely generalizes the results of [17] from Hilbert spaces to Banach spaces.

Remark 3.7. Theorem 3.5 improves Theorem 1.2 in the following senses.

(1) The inverse-strong-monotonicity of $A$ is relaxed to monotonicity and Lipschitz continuity.

(2) The assumption (A) is removed.

Remark 3.8. Remarks 3.6 and 3.7 show Theorem 3.5 generalizes Theorem 1.5 from Hilbert spaces to Banach spaces and solves simultaneously problems (P1) and (P2) without additional assumptions. 
Remark 3.9. The statement (on convergence) similar to Theorem 3.5 will also take place for the following iterative process:

$$
\left\{\begin{array}{l}
x_{n+1}=\Pi_{H_{n}} J^{-1}\left(J x_{n}-\lambda_{n} A\left(y_{n}\right)\right) \\
y_{n+1}=\Pi_{C} J^{-1}\left(J x_{n+1}-\lambda_{n} A\left(y_{n}\right)\right)
\end{array}\right.
$$

where $H_{n}=\left\{z \in E:\left\langle z-y_{n}, J x_{n}-\lambda_{n} A y_{n-1}-J y_{n}\right\rangle \leqslant 0\right\}$, provided that $0<\inf _{n} \lambda_{n} \leqslant \sup _{n} \lambda_{n}<\frac{c_{1}}{3 L}$.

\section{Numerical experiments}

In this section, we give two numerical examples to support our theoretical results.

Example 4.1. Let $E=R^{2}$ with the standard inner product $\langle\vec{x}, \vec{y}\rangle=\vec{x}^{\top} \vec{y}$ and the induced norm $\|\vec{x}\|=$ $\sqrt{\langle\vec{x}, \vec{x}\rangle}$ for all $\vec{x}, \vec{y} \in R^{2}$, where $\vec{x}^{\top}$ denotes the transposition of $\vec{x}$. Let $C=\left\{(x, y)^{\top} \in R^{2} \mid x \geqslant 0, y \geqslant 0\right\}$ and $A: C \rightarrow R^{2}$ be defined by

$$
A(\vec{x}):=M \vec{x}, \forall \vec{x} \in C,
$$

where

$$
M=\left(\begin{array}{cc}
0 & -1 \\
1 & 0
\end{array}\right) \text { is a matrix. }
$$

A simple computation shows

$$
\langle A(\vec{x})-A(\vec{y}), \vec{x}-\vec{y}\rangle=\langle M \vec{x}-M \vec{y}, \vec{x}-\vec{y}\rangle=\langle M(\vec{x}-\vec{y}), \vec{x}-\vec{y}\rangle=0,
$$

and

$$
\|A(\vec{x})-A(\vec{y})\|^{2}=\|M(\vec{x}-\vec{y})\|^{2}=\|\vec{x}-\vec{y}\|^{2}, \forall \vec{x}, \vec{y} \in C .
$$

It follows from (4.1) and (4.2) that $A$ is monotone and 1-Lipschitz continuous and $A$ is not inverselystrongly monotone. It is also clear that $\operatorname{VI}(C, A)=\left\{(x, 0)^{\top} \in R^{2} \mid x \geqslant 0\right\}$, i.e., $\operatorname{VI}(C, A)$ is the positive horizontal axis. In addition, taking $\vec{y}=(2,1)^{\top} \in C$ and $\vec{u}=(1,0)^{\top} \in \operatorname{VI}(C, A)$, we have

$$
\|A(\vec{y})\|=\|M \vec{y}\|=\sqrt{5} \geqslant\|A(\vec{y})-A(\vec{u})\|=\sqrt{2},
$$

which implies that the example does not satisfy the condition (A) of Section 1. Hence, Algorithm 1.1 is invalid for the example. However, we can apply Algorithm 1.3 constructed by [16] and Algorithm 3.1 of this paper to the example. In the following, we illustrate the numerical experiment results to compare Algorithm 1.3 constructed in [16] with Algorithm 3.1.

In the following experiments, we always take $\lambda=\frac{1}{4}$ and $\alpha_{n}=\frac{1}{n}$. It is easy to see that the conditions on $\lambda$ and $\left\{\alpha_{n}\right\}$ are satisfied. We denote the time of execution in second by (CPU(s)).

Now, we take the initial points $\overrightarrow{x_{0}}=\overrightarrow{y_{0}}=(0,1)^{\mathrm{T}}$. Then, the sequence $\left\{x_{n}\right\}$ generated by Algorithm 3.1 converges strongly to an element of $\operatorname{VI}(C, A)$. We list the operating results of Algorithm 3.1 in Table 1.

Table 1: The operating results of Algorithm 3.1.

\begin{tabular}{|c|c|c|c|}
\hline \multirow{2}{*}{$\mathrm{n}$} & \multicolumn{3}{|c|}{ initial values $\overrightarrow{\mathrm{x}_{0}}=\overrightarrow{\mathrm{y}_{0}}=(0,1)^{\top}$} \\
\cline { 2 - 4 } & $\overrightarrow{\mathrm{x}_{\mathrm{n}}}$ & $\overrightarrow{\mathrm{y}_{\mathrm{n}}}$ & $\mathrm{CPU}(\mathrm{s})$ \\
\hline 1 & $(0.25,1)^{\top}$ & $(0.5,1)^{\top}$ & $3.377614 \mathrm{e}-04$ \\
\hline 2 & $(0.5,0.875)^{\top}$ & $(0.75,0.75)^{\top}$ & $4.128713 \mathrm{e}-04$ \\
\hline 3 & $(0.6875,0.6875)^{\top}$ & $(0.875,0.5)^{\top}$ & $4.438262 \mathrm{e}-04$ \\
\hline 4 & $(0.8125,0.4688)^{\top}$ & $(0.9375,0.25)^{\top}$ & $4.489579 \mathrm{e}-04$ \\
\hline 5 & $(0.875,0.2344)^{\top}$ & $(0.9375,0)^{\top}$ & $4.900118 \mathrm{e}-04$ \\
\hline 6 & $(0.875,0)^{\top}$ & $(0.875,0)^{\top}$ & $5.128713 \mathrm{e}-04$ \\
\hline 7 & $(0.875,0)^{\top}$ & $(0.875,0)^{\top}$ & $5.296661 \mathrm{e}-04$ \\
\hline
\end{tabular}


Remark 4.2. From Table 1, we can see that if we take the initial values $\overrightarrow{x_{0}}=\overrightarrow{y_{0}}=(0,1)^{\top}$, Algorithm 3.1 executes a finite steps and we can obtain a solution $(0.875,0)^{\top} \in \operatorname{VI}(C, A)$ and the time of execution is 5.296661e-04 second.

Next, we list the operating results of Algorithm 1.3 when the initial point $\overrightarrow{x_{1}}=(0,1)^{\top}$. The stopping criteria is $\left\|\overrightarrow{x_{n}}-\overrightarrow{y_{n}}\right\| \leqslant 10^{-6}$.

Table 2: The operating results of Algorithm 1.3.

\begin{tabular}{|c|c|c|c|}
\hline \multirow{2}{*}{$n$} & \multicolumn{3}{|c|}{ initial value $\overrightarrow{x_{1}}=(0,1)^{\top}$} \\
\cline { 2 - 4 } & $\overrightarrow{x_{n}}$ & $\overrightarrow{y_{n}}$ & CPU(s) \\
\hline 3 & $(0.125000,0.968750)^{\top}$ & $(0.367188,0.937500)^{\top}$ & $3.853466 \mathrm{e}-04$ \\
\hline 100 & $(0.063856,0.010000)^{\mathrm{T}}$ & $(0.066356,0.000000)^{\mathrm{T}}$ & $1.292730 \mathrm{e}-03$ \\
\hline 1000 & $(0.006386,0.001000)^{\mathrm{T}}$ & $(0.006636,0.000000)^{\mathrm{T}}$ & $8.819585 \mathrm{e}-03$ \\
\hline 10000 & $(0.000639,0.000100)^{\mathrm{T}}$ & $(0.000664,0.000000)^{\mathrm{T}}$ & $5.886985 \mathrm{e}-02$ \\
\hline 100000 & $(0.000064,0.0000010)^{\mathrm{T}}$ & $(0.000066,0.000000)^{\mathrm{T}}$ & $5.369086 \mathrm{e}-01$ \\
\hline 500000 & $(0.000013,0.000002)^{\mathrm{T}}$ & $(0.000013,0.000000)^{\mathrm{T}}$ & $2.661354 \mathrm{e}+00$ \\
\hline 1030777 & $(0.000006,0.000001)^{\mathrm{T}}$ & $(0.000006,0.000000)^{\mathrm{T}}$ & $1.280020 \mathrm{e}+01$ \\
\hline
\end{tabular}

Remark 4.3. From Table 2, we can see that if we take the initial value $\overrightarrow{x_{1}}=(0,1)^{\top}$, Algorithm 1.3 executes an infinite steps and we can obtain an approximative solution $(0.000006,0.000000)^{\top}$ for the variational inequality (1.1) in the example and the time of execution is $1.280020 \mathrm{e}+01$ second.

Remark 4.4. From Tables 1 and 2, we see that our algorithm (Algorithm 3.1) has a competitive advantage over the time of execution in second (CPU(s)) and the iterative number being far less than those of Algorithm 1.3 constructed in [16] provided with the same initial point.

Example 4.5. Let $E=\mathbb{R}$ with the standard inner product $\langle x, y\rangle=x y$ and the induced norm $\|x\|=|x|$ for all $x, y \in \mathbb{R}$. Let $C=\left[-\frac{\pi}{2}, \frac{\pi}{2}\right]$ and $A: C \rightarrow \mathbb{R}$ be defined by $A(x):=\sin (x)$ for all $x \in C$. We can easily see that $A$ is monotone and 1-Lipschitz continuous. Furthermore, $\operatorname{VI}(C, A)=\{0\}$.

In the following experiments, we take $\lambda=\frac{1}{4}$ and $\alpha_{n}=\frac{1}{n}$. It is easy to see that the conditions on $\lambda$ and $\left\{\alpha_{n}\right\}$ are satisfied.

By Algorithm 3.1, we obtain

$$
\left\{\begin{array}{l}
x_{2}=P_{C}\left[x_{1}-\frac{1}{4} \sin \left(y_{1}\right)\right] \\
y_{2}=P_{C}\left[x_{2}-\frac{1}{4} \sin \left(y_{1}\right)\right], \\
H_{n}:=\left\{z \in \mathbb{R} \mid\left\langle z-y_{n}, x_{n}-\frac{1}{4} \sin \left(y_{n-1}\right)-y_{n}\right\rangle \leqslant 0\right\}, n \geqslant 2, \\
x_{n+1}=P_{H_{n}}\left[x_{n}-\frac{1}{4} \sin \left(y_{n}\right)\right], \\
y_{n+1}=P_{C}\left[x_{n+1}-\frac{1}{4} \sin \left(y_{n}\right)\right] .
\end{array}\right.
$$

Again, by Algorithm 1.3, we obtain

$$
\left\{\begin{array}{l}
u_{n}=P_{C}\left[x_{n}-\frac{1}{4} \sin \left(x_{n}\right)\right], \\
T_{n}:=\left\{w \in \mathbb{R}:\left\langle w-u_{n}, x_{n}-\frac{1}{4} \sin \left(x_{n}\right)-u_{n}\right\rangle \leqslant 0\right\} \\
z_{n}=P_{T_{n}}\left[x_{n}-\frac{1}{4} \sin \left(u_{n}\right)\right] \\
x_{n+1}=\frac{1}{n} \times x_{1}+\left(1-\frac{1}{n}\right) z_{n} .
\end{array}\right.
$$

Then, the sequences $\left\{x_{n}\right\}$ generated by (4.3) and (4.4) converge strongly to $x^{*}=0 \in \operatorname{VI}(C, A)$.

Now, we take the initial points $x_{1}=y_{1}=\frac{\pi}{2}, \quad x_{1}=y_{1}=\frac{\pi}{3}, \quad x_{1}=y_{1}=\frac{\pi}{4}$, respectively. By the above recursion formulas (4.3) and (4.4), we illustrate the numerical experiment results of Algorithm 3.1 and Algorithm 1.3 in Table 3 to compare the time of execution in second (CPU(s)) and the number of iterations (Iter.). The stopping criteria is $\left\|x_{n}-x^{*}\right\|=\left\|x_{n}\right\|=\left|x_{n}\right| \leqslant T O L=10^{-6}$. 
Table 3: Comparison of Algorithms 3.1 and 1.3.

\begin{tabular}{|c|c|c|c|c|c|}
\hline \multirow{2}{*}{$x_{1}$} & \multirow{2}{*}{ TOL } & \multicolumn{2}{|c|}{ Algorithms 3.1 } & \multicolumn{2}{c|}{ Algorithms 1.3} \\
\cline { 3 - 6 } & CPU(s) & Iter. & CPU(s) & Iter. \\
\hline$\frac{\pi}{2}$ & $10^{-6}$ & $1.026356 \mathrm{e}-05$ & 68 & $1.164914 \mathrm{e}-01$ & 866647 \\
\hline$\frac{\pi}{3}$ & $10^{-6}$ & $6.997884 \mathrm{e}-06$ & 66 & $4.991638 \mathrm{e}-02$ & 577765 \\
\hline$\frac{\pi}{4}$ & $10^{-6}$ & $3.265679 \mathrm{e}-06$ & 64 & $2.945176 \mathrm{e}-02$ & 433324 \\
\hline
\end{tabular}

Remark 4.6. From Table 3, we also see that our algorithm (Algorithm 3.1) has a competitive advantage over the time of execution in second (CPU(s)) and the iterative number being far less than those of Algorithm 1.3 provided with the same initial point and tolerance.

Remark 4.7. All the programs are written in Matlab 7.0. The experiments are performed on a PC Intel(R) Core (TM) i5-5200U CPU @ 2.20GHz 2.20GHz, RAM 4.00GB.

\section{Conclusions}

We modify the extragradient algorithm introduced by Malitsky and Semenov in [17] and construct Algorithm 3.1 to find an element of $\mathrm{VI}(\mathrm{C}, \mathrm{A})$ for a Lipschitz-continuous, monotone mapping in a 2-uniformly convex and uniformly smooth Banach space. The weak convergence of this Algorithm is proved. Consequently, we generalize the results of [17] from Hilbert spaces to Banach spaces and solve simultaneously problems (P1) and (P2). Furthermore, we give two simple examples which show Algorithm 3.1 constructed by this paper has a competitive advantage over the time of execution and the iterative number being far less than those of Algorithm 1.3 constructed in [16] provided with the same initial point. However, Algorithm 3.1 has only weak convergence which is the weakness of the algorithm. In order to overcome this weakness, we can not rely only on the process itself; instead, some additional step of iteration should be taken. As did in [13], the authors combined the subgradient extragradient method (1.4) with the Halpern method to obtain the strong convergence, which is a inspiration to improve Algorithm 3.1 for strong convergence in the future research.

\section{Acknowledgment}

We would like to thank the editor and anonymous referees for their valuable comments and suggestions, which helped us very much in improving the paper. This work was financially supported by the National Natural Science Foundation of China (11401157).

\section{References}

[1] Y. I. Al'ber, S. Reich, An iterative method for solving a class of nonlinear operator equations in Banach spaces, Panamer. Math. J., 4 (1994), 39-54. 2, 2, 2

[2] K. Ball, E. A. Carlen, E. H. Lieb, Sharp uniform convexity and smoothness inequalities for trace norms, Invent. Math., 115 (1994), 463-482. 2

[3] N. Buong, Strong convergence theorem of an iterative method for variational inequalities and fixed point problems in Hilbert spaces, Appl. Math. Comput., 217 (2010), 322-329. 1

[4] L.-C. Ceng, N. Hadjisavvas, N.-C. Wong, Strong convergence theorem by a hybrid extragradient-like approximation method for variational inequalities and fixed point problems, J. Global Optim., 46 (2010), 635-646. 1, 1

[5] Y. Censor, A. Gibali, S. Reich, Strong convergence of subgradient extragradient methods for the variational inequality problem in Hilbert space, Optim. Methods Softw., 26 (2011), 827-845. 1

[6] Y. Censor, A. Gibali, S. Reich, The subgradient extragradient method for solving variational inequalities in Hilbert space, J. Optim. Theory Appl., 148 (2011), 318-335. 1, 1, 1, 1

[7] J.-M. Chen, L.-J. Zhang, T.-G. Fan, Viscosity approximation methods for nonexpansive mappings and monotone mappings, J. Math. Anal. Appl., 334 (2007), 1450-1461. 1

[8] C.-J. Fang, Y. Wang, S.-K. Yang, Two algorithms for solving single-valued variational inequalities and fixed point problems, J. Fixed Point Theory Appl., 18 (2016), 27-43. 1 
[9] H. Iiduka, W. Takahashi, Strong convergence theorems for nonexpansive nonself-mappings and inverse-strongly-monotone mappings, J. Convex Anal., 11 (2004), 69-79. 1

[10] H. Iiduka, W. Takahashi, Strong convergence theorems for nonexpansive mappings and inverse-strongly monotone mappings, Nonlinear Anal., 61 (2005), 341-350.

[11] H. Iiduka, W. Takahashi, Weak convergence of a projection algorithm for variational inequalities in a Banach space, J. Math. Anal. Appl., 339 (2008), 668-679. 1, 1, 2.2, 2.3, 2.4, 3

[12] G. M. Korpelevič, An extragradient method for finding saddle points and for other problems, (Russian) Ékonom. i Mat. Metody, 12 (1976), 747-756. 1

[13] R. Kraikaew, S. Saejung, Strong convergence of the Halpern subgradient extragradient method for solving variational inequalities in Hilbert spaces, J. Optim. Theory Appl., 163 (2014), 399-412. 1, 1, 1, 5

[14] J.-L. Lions, Optimal control of systems governed by partial differential equations, Translated from the French by S. K. Mitter, Die Grundlehren der mathematischen Wissenschaften, Band 170 Springer-Verlag, New York-Berlin, (1971). 1

[15] Y. Liu, Strong convergence theorem for relatively nonexpansive mapping and inverse-strongly-monotone mapping in a Banach space, Appl. Math. Mech. (English Ed.), 30 (2009), 925-932. 1

[16] Y. Liu, Strong convergence of the Halpern subgradient extragradient method for solving variational inequalities in Banach spaces, J. Nonlinear Sci. Appl., 10 (2017), 395-409. 1, 1.3, 4.1, 4.4, 5

[17] Y. V. Malitsky, V. V. Semenov, An extragradient algorithm for monotone variational inequalities, Translation of Kibernet. Sistem. Anal., 2014 (2014), 125-131, Cybernet. Systems Anal., 50 (2014), 271-277. 1, 3, 3.6, 5

[18] S.-Y. Matsushita, W. Takahashi, A strong convergence theorem for relatively nonexpansive mappings in a Banach space, J. Approx. Theory, 134 (2005), 257-266. 2.1, 2.2, 2.3

[19] N. Nadezhkina, W. Takahashi, Strong convergence theorem by a hybrid method for nonexpansive mappings and Lipschitzcontinuous monotone mappings, SIAM J. Optim., 16 (2006), 1230-1241. 1, 1

[20] K. Nakajo, Strong convergence for gradient projection method and relatively nonexpansive mappings in Banach spaces, Appl. Math. Comput., 271 (2015), 251-258. 1, 2.5

[21] L. D. Popov, A modification of the Arrow-Hurwitz method of search for saddle points, (Russian) Mat. Zametki, 28 (1980), 777-784. 1

[22] Y. Takahashi, K. Hashimoto, M. Kato, On sharp uniform convexity, smoothness, and strong type, cotype inequalities, J. Nonlinear Convex Anal., 3 (2002), 267-281. 2

[23] W. Takahashi, M. Toyoda, Weak convergence theorems for nonexpansive mappings and monotone mappings, J. Optim. Theory Appl., 118 (2003), 417-428. 1

[24] K.-K. Tan, H. K. Xu, Approximating fixed points of nonexpansive mappings by the Ishikawa iteration process, J. Math. Anal. Appl., 178 (1993), 301-308. 2.6

[25] A. R. Tufa, H. Zegeye, An algorithm for finding a common point of the solutions of fixed point and variational inequality problems in Banach spaces, Arab. J. Math. (Springer), 4 (2015), 199-213. 1 